\title{
Characterization of single-nucleotide variation in Indian-origin rhesus macaques (Macaca mulatta)
}

Gloria L Fawcett ${ }^{1,2}$, Muthuswamy Raveendran', David Rio Deiros ${ }^{1}$, David Chen ${ }^{1}$, Fuli Yu ${ }^{1,2}$, Ronald Alan Harris ${ }^{2}$, Yanru Ren ${ }^{1}$, Donna M Muzny ${ }^{1,2}$, Jeffrey G Reid ${ }^{1,2}$, David A Wheeler ${ }^{1,2}$, Kimberly C Worley ${ }^{1,2}$, Steven E Shelton ${ }^{4}$, Ned H Kalin ${ }^{4,5}$, Aleksandar Milosavljevic ${ }^{2}$, Richard Gibbs ${ }^{1,2}$ and Jeffrey Rogers ${ }^{1,2,3^{*}}$

\begin{abstract}
Background: Rhesus macaques are the most widely utilized nonhuman primate model in biomedical research. Previous efforts have validated fewer than 900 single nucleotide polymorphisms (SNPs) in this species, which limits opportunities for genetic studies related to health and disease. Extensive information about SNPs and other genetic variation in rhesus macaques would facilitate valuable genetic analyses, as well as provide markers for genome-wide linkage analysis and the genetic management of captive breeding colonies.

Results: We used the available rhesus macaque draft genome sequence, new sequence data from unrelated individuals and existing published sequence data to create a genome-wide SNP resource for Indian-origin rhesus monkeys. The original reference animal and two additional Indian-origin individuals were resequenced to low coverage using SOLiDTM sequencing. We then used three strategies to validate SNPS: comparison of potential SNPs found in the same individual using two different sequencing chemistries, and comparison of potential SNPs in different individuals identified with either the same or different sequencing chemistries. Our approach validated approximately 3 million SNPs distributed across the genome. Preliminary analysis of SNP annotations suggests that a substantial number of these macaque SNPs may have functional effects. More than 700 non-synonymous SNPs were scored by Polyphen-2 as either possibly or probably damaging to protein function and these variants now constitute potential models for studying functional genetic variation relevant to human physiology and disease.

Conclusions: Resequencing of a small number of animals identified greater than 3 million SNPs. This provides a significant new information resource for rhesus macaques, an important research animal. The data also suggests that overall genetic variation is high in this species. We identified many potentially damaging non-synonymous coding SNPs, providing new opportunities to identify rhesus models for human disease.
\end{abstract}

Keywords: single nucleotide polymorphism, common variants, SOLiD ${ }^{\mathrm{TM}}$, genetic variation, rhesus macaque

\section{Background}

Experimental study of rhesus macaques (Macaca mulatta) is critical to progress in many aspects of biomedical research. This species is widely used in neurobiology and behavioral science [1-5], endocrinology [6,7], physiological studies of cardiovascular disease and obesity [8], diabetes [9], alcoholism and addiction research $[10,11]$, and other diverse fields $[12,13]$. Rhesus macaques are central to progress in the development of

\footnotetext{
* Correspondence: jr13@bcm.edu

'Human Genome Sequencing Center, Department of Molecular and Human Genetics, Baylor College of Medicine, Houston, Texas 77030, USA

Full list of author information is available at the end of the article
}

vaccines and anti-viral therapies against HIV infection and AIDS because these animals are the best available animal model for studies of lentivirus infection [14,15]. However, despite the wide use of rhesus macaques in research, this species is under-utilized as a model organism for investigation of genetic processes influencing human biology and human disease. One factor limiting the use of rhesus monkeys in genetic analyses is the lack of existing information about intra-species polymorphism. Currently, dbSNP contains fewer than 900 validated SNPs for this species (http://www.ncbi.nlm.nih. gov/projects/SNP/) [16] and only a small number of copy number variants (CNVs) have been described

\section{Biomed Central}


$[17,18]$. Clearly, more information about genetic variation segregating within this otherwise widely studied species would be useful.

Additional effort to identify both common and low frequency genetic variation in this species is warranted, based on the potential opportunity for and significance of developing new genetic models of human disease. Despite the limited information available today, a number of studies have shown that rhesus monkeys exhibit intra-species genetic variation in the same genes that have been associated with risk of disease in humans. Furthermore, recent analyses have demonstrated that DNA sequence variation among rhesus macaques can parallel the functional effects of known variation in humans, providing remarkably similar and thus highly informative animal models of human genetic risk. In one example, researchers found natural mutations segregating among captive rhesus monkeys in two genes that had previously been associated with macular degeneration in humans [19]. The investigators found that, as in humans, the polymorphisms in macaques were associated with individual variation in the major risk factor for macular degeneration, demonstrating a parallel genetic effect in rhesus and establishing this as an important model of genetic predisposition to this disease. Other examples of genetic variation among rhesus macaques that parallels human functional variation are known in the serotonin transporter $[20,21]$ and the muopioid receptor [22].

Based on these observations, we anticipated that genome-wide knowledge of SNP variation segregating within research colonies of Indian-origin rhesus macaques would produce novel and valuable information about functionally significant genetic variation in a number of important cellular pathways. Broad knowledge of both common and low frequency sequence variation in rhesus macaques (and by implication, equivalent information for other species of nonhuman primates) will facilitate discovery of new animal models of functional variation in specific genetic pathways and thus help investigators to elucidate mechanisms associated with human disease [17]. It is important to recognize that nonhuman primates constitute a unique set of animal models that offer a singular combination of research advantages. The overall genetic and physiological similarity of macaques and other nonhuman primates to humans $[3,23]$ coupled with the opportunity to examine sets of animals raised under controlled environmental circumstances, or subject to precise environmental manipulations (e.g. exposure to therapeutic drugs or changes in diet), creates outstanding research opportunities. Analyses in macaques can examine the specific effects of individual genetic variants on those cellular pathways, the effects of genotype-by-genotype or genotype-by-environment interaction, or other complex processes that are relevant to human disease but cannot be readily examined in human subjects under such tightly controlled environments.

Unfortunately, little is known about genetic variation in rhesus macaques. Fewer than 900 validated SNPs and 100 CNVs have been described. The available data [23-28] suggest that a given number of rhesus macaques will show as much or more diversity and heterozygosity as the same number of humans. We undertook this study to assess the value of whole genome re-sequencing in rhesus macaques for the discovery of novel SNPs. We identified and bioinformatically validated SNPs throughout the rhesus macaque genome using existing sequence data and new SOLiD sequencing reads we generated from three unrelated macaques. We are particularly interested in the identification of novel variation among these animals that has potentially significant effects on gene function and physiological processes relevant to human disease.

\section{Results}

We identified and validated more than 3 million unique SNPs in rhesus macaques through pairwise comparisons of SNP data sets (Table 1). Our starting point for these analyses was a list of 4.3 million inferred heterozygous positions in the original Sanger sequencing data for the reference animal [23]. Comparison of these Sanger data with corona_lite SNP calls from SOLiD re-sequencing of the reference animal resulted in validation of 1,056,266 heterozygous positions (Figure 1). Similarly, re-sequencing of two additional Indian-origin animals, followed by comparison to the reference animal SNP list, resulted in the validation of an additional 873,552 SNPs.

We were able to validate 1,058,581 additional SNPs among just three rhesus macaques using e-genotyping (http://is04607.com/ drio/egenotype/[29]). E-genotyping is a novel in silico method to survey mapped read sequence data using $31 \mathrm{bp}$ probe sequences created from a list of known or suspected SNPs. Unvalidated potential heterozygous positions from the original Sanger reference animal data were used to create uniquely mapping e-genotyping probes. Using these probes we screened each of the SOLiD read sets (i.e. the reference animal and the two additional animals). This identified 527,425 validated SNPs that were not validated using the Sanger data and corona_lite analyses of SOLiD data. Probes created from the corona_lite analyses of SOLiD data were used to identify and validate 531,156 more SNPs.

Finally, we used the smaller published datasets to complete a comprehensive list of validated SNPs. We validated 49,747 SNPs by comparing the results from the corona_lite re-sequencing analyses with previously published datasets. A small number of SNPs $(n=20)$ 
Table 1 Bioinformatically validated SNPs

\begin{tabular}{|c|c|c|c|c|c|c|c|c|}
\hline & 17573 Sanger & 17573 SOLiD & $r 1766$ & r02120 & Sub-species comparison & MamuSNP & ENCODE & $\mathrm{dbSNP}$ \\
\hline \multicolumn{9}{|l|}{17573 Sanger } \\
\hline 17573 SOLiD & $1,056,266$ & & & & & & & \\
\hline r1766 & 22,665 & 412,920 & & & & & & \\
\hline $\mathrm{r} 02120$ & 5,734 & 103,323 & 328,910 & & & & & \\
\hline Sub-species comparison & 4,185 & 6,519 & 1,070 & 226 & & & & \\
\hline MamuSNP & 389 & 781 & 159 & 33 & 19 & & & \\
\hline ENCODE & 29 & 76 & 14 & 7 & 0 & 1 & & \\
\hline $\mathrm{dbSNP}$ & 11 & 12 & 5 & 0 & 0 & 0 & 0 & \\
\hline egeno.assembly.hets & - & 421,234 & 64,584 & 41,607 & 34,339 & - & - & - \\
\hline egeno.17573.SOLiD & - & - & 109,485 & 61,941 & 9 & - & - & - \\
\hline egeno.r1766.frag & - & 155,454 & - & 93,016 & 17 & - & - & - \\
\hline egeno.r02120.frag & - & 41,836 & 69,424 & - & 3 & - & - & - \\
\hline egeno.sub-species comp & - & 677 & 743 & 443 & - & - & - & - \\
\hline
\end{tabular}

Unique non-overlapping validated SNPs identified in each pair-wise comparison are displayed. Validated SNPs were determined on the basis of both alleles at a genomic location being identified in at least two data sets and one of either multiple animals or multiple chemistries. Validated SNPs that fit into multiple cells were represented in the left-most appropriate cell.

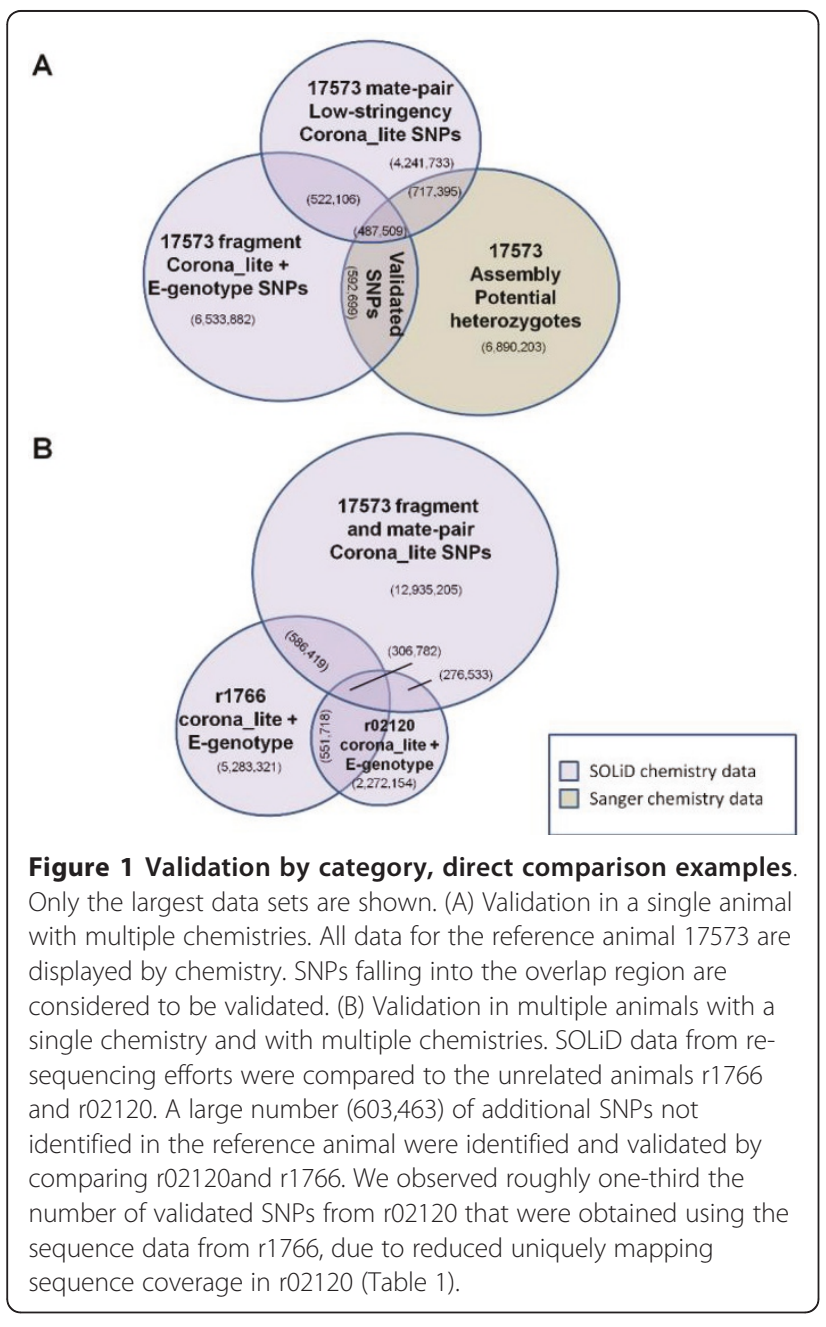

were validated through comparisons among the previously published data. These 20 SNPs were not found in our new SOLiD re-sequencing data (Figure S1, Additional file 1).

The genomic distribution of the validated SNPs appears to be random across the autosomes (Figure 2). SNP distribution by chromosome was consistent with chromosome length, except for the X chromosome. X chromosome SNP discovery is limited in this study because one of the three study animals was male (r02120). Across the autosomes, the density of SNPs per $\mathrm{Mb}$ fits within one standard deviation (195.3 SNPs/Mb) from the overall means $(1,057.1 \mathrm{SNPs} / \mathrm{Mb})$. We retained lists of the unvalidated SNPs in order to facilitate future efforts at SNP discovery in genomic/genic regions where coverage is relatively sparse. This data is provided in our Genboree website (http://genboree.org/java-bin/project. jsp? projectName $=$ Rhesus\%20SNPs\%20using\%20NextGen\%20Sequencing\&isPublic $=\mathrm{Yes}[30]$ ) in the "Rhesus SNPs" database in a track called "Unvalidated:SNPs".

Unidentified nearly identical duplications of sequence (e.g. unrecognized segmental duplications) in the rhesus whole genome assembly are one potential cause of false positive SNPs. If nearly identical duplications were mistakenly collapsed in the genome assembly, then wherever there are single base differences between two copies of a sequence, the reference animal would appear heterozygous for a SNP. The other two animals would also appear heterozygous for the same SNP, thus providing false validation. We addressed this issue in several ways. First, we note that this problem would produce false heterozygote calls across all three animals, and we 


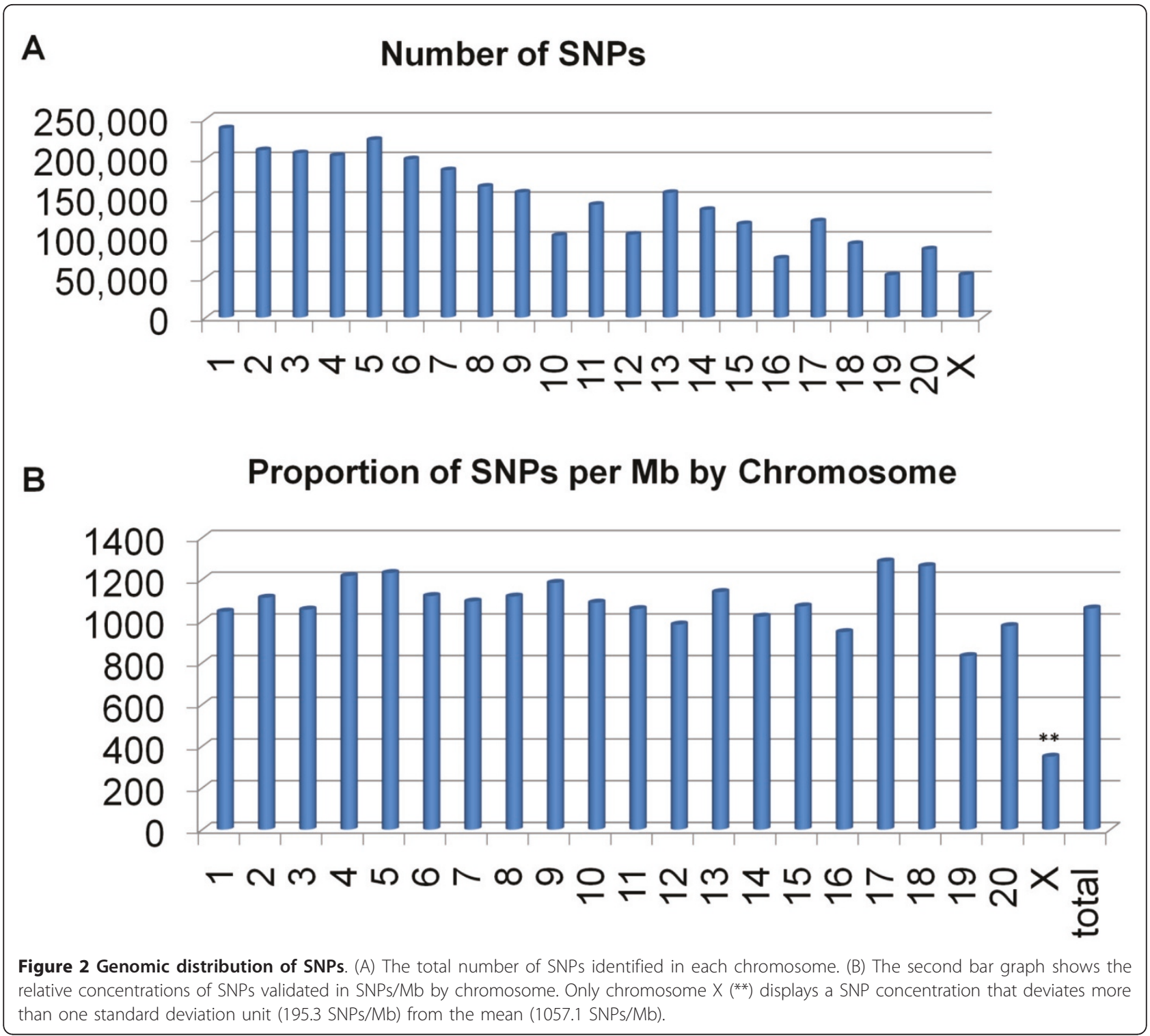

found that only $\sim 10 \%$ of all of our validated SNPs are scored as heterozygous in all three animals (note however, that the animal with the lowest coverage, r02120, limits the number of SNPs identified as heterozygous in all sequenced animals). Among the 306,782 SNPs that are heterozygous in all subjects, we can evaluate the impact of cryptic sequence duplication in two ways. We compared Sanger read coverage for these 306,782 SNPs against an equivalent number of SNPs that were scored as heterozygous in two animals and homozygous in one animal. Unrecognized duplications should have higher than average read coverage in the original $5.2 \times$ Sanger whole genome read data. We found (Figure S2, Additional file 2) that the mean coverage and read coverage distributions for the two sets of SNPs is virtually identical. If a substantial fraction of the "three heterozygote"
SNPs were false positives due to unrecognized duplications in the genome assembly, we would expect a higher mean read coverage for that set compared with the control SNPs that are not scored as heterozygous in all three animals. The percentage of SNPs with read coverage of 11 or greater (i.e. twice the genome-wide average) was only $3.8 \%$ for SNPs detected in all three individuals and $3.5 \%$ for SNPs detected in only two individuals $(0.38 \%$ and $0.35 \%$ respectively of all of our validated SNPs).

An alternative hypothesis is that duplicated sequences within the rhesus assembly were not assembled in the chromosome scaffolds and were instead placed in the "chromosome unknown" or chrUr bin. The tendency for duplications to be included in unknown chromosomes has been noted in other whole genome shotgun 
assemblies [31]. Therefore we wished to determine whether our validated SNPs had homology to assembled contigs placed in chrUr, which might represent unrecognized duplications. To test this, we split chrUr into 150 bp length "reads" and scanned this simulated "read" data using e-genotype probes created from all of our validated rhesus SNPs $(3,271,622$ probes, representing the reference position at all locations as well as the one or two non-reference validated alleles). None of the probes matched sequence from the unmapped reference sequence provided in chrUr. Given these various results, we conclude that there is no clear evidence for a significant number of false positive SNP calls due to duplicated genomic regions in our SNP data.

\section{SNP annotation and Polyphen Analysis}

All the validated SNPs were further examined to characterize their position relative to known coding regions and possible functionality (Figure 3 ). Due to the incomplete annotation of the rhesus reference genome, the annotations of rhesus SNPs are based in part on mapping to homologous human transcripts. As expected,

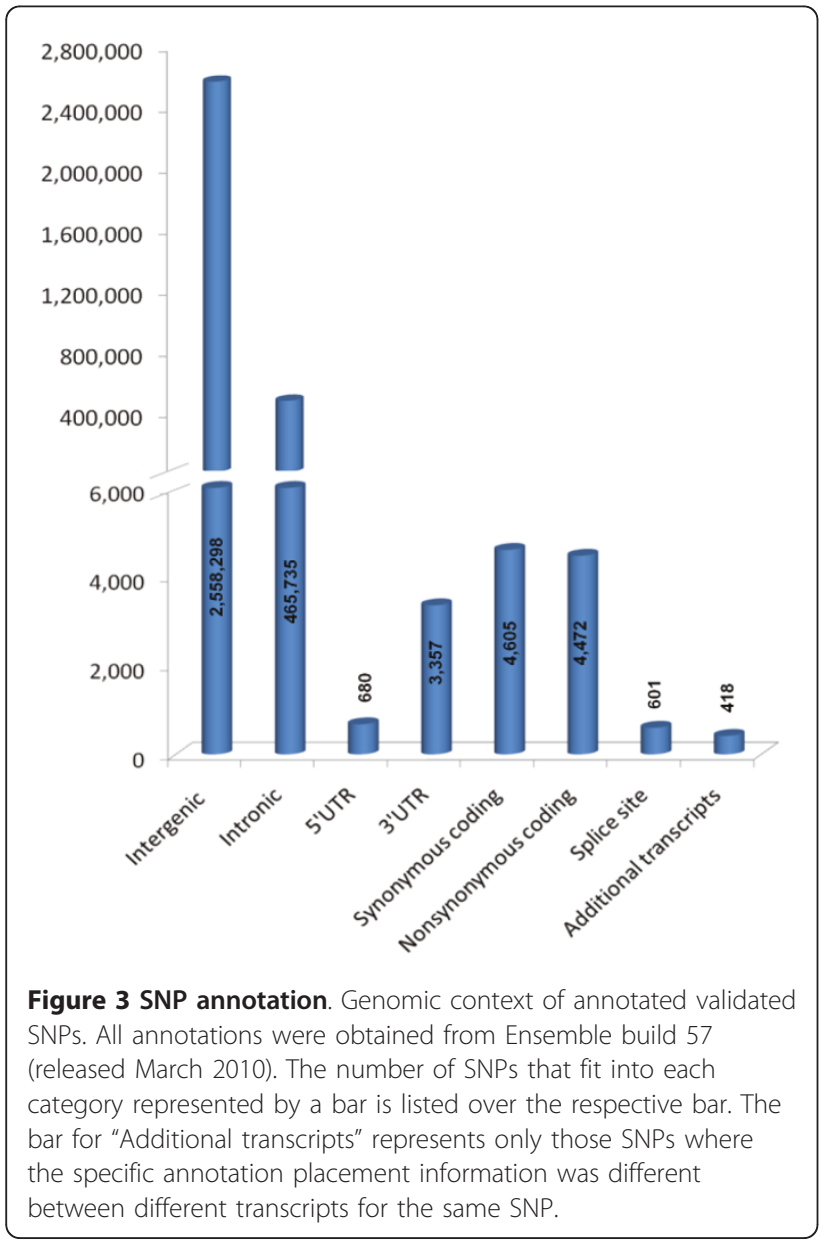

most validated SNPs map to intergenic regions $(84.2 \%$ of validated SNPs). Intronic SNPs were the second most prevalent at $15.3 \%$. Very few (601 or $0.02 \%$ ) of the SNPs fell into known or predicted splice sites. Among coding SNPs, about equal numbers are synonymous changes $(4,605,0.15 \%$ of total) and non-synonymous $(4,472$, $0.15 \%$ of total). A number of SNPs fell into regions of alternative splicing such that the genomic placement of the SNP varied by transcript. All such SNPs $(n=418)$ are associated with a coding sequence of at least one transcript, and with an alternative transcript where the placement differed: synonymous coding (21.5\%), nonsynonymous coding (19.1\%), 5'UTR (28.9\%), 3'UTR (30.4\%).

Potentially functional polymorphisms in rhesus macaques are of particular interest, especially those in genes that may correlate with human disease. The rhesus genome annotation is incomplete and based primarily upon gene prediction algorithms that rely upon human-torhesus homology [32-34]. Therefore, we relied upon a homology-based method (see Methods) to generate hypotheses about the functionality of non-synonymous SNPs (nsSNPs) (Table 2). A total of 4,439 SNPs from the full list of nsSNPs $(4,472)$ were successfully converted from rheMac2 coordinates to hg18 coordinates. After removing all SNPs where the hg18 reference allele matched the new variant macaque allele, 4,177 nsSNPs were submitted to Polyphen-2 (http://genetics.bwh.harvard.edu/pph2/[35]) [36]. Of those, 411 were scored as probably damaging and 325 scored as possibly damaging. SNPs that affect binding sites or are located in transmembrane regions can often be expected to alter protein function. Twelve of the probably damaging mutations are annotated as falling in transmembrane regions, two affect a metal binding site, and one affects a modified residue. Of the possibly damaging mutations, six are in transmembrane regions, one is in a propeptide, two affect modified residues, and one affects a carbohydrate binding site. We performed GeneGo (http:// www.genego.com/[37]) pathway analysis to determine which diseases were associated with the genes containing predicted deleterious rhesus SNPs from the PolyPhen-2 analysis. These genes were involved in one or more critical organism process such as apoptotic pathways, DNA repair, development or inflammation. Table 2 displays a non-exhaustive list of diseases associated with the relevant pathways, many of which are due to a few well-known genes: Brca2, Ppgca1b, Map3k1, and Casp7.

Finally, we compared the list of validated Indian-origin rhesus macaque SNPs to the locations of known SNPs in the human genome (dbSNP, build 132). The evolutionary lineage leading to rhesus macaques diverged from the human lineage about 22-26 million years ago, 
Table 2 Diseases associated with genes containing nsSNPs

\begin{tabular}{|c|c|c|c|c|c|}
\hline $\begin{array}{l}\text { General } \\
\text { Category }\end{array}$ & $\begin{array}{c}\text { \# Associated } \\
\text { Deleterious SNPs }\end{array}$ & $\begin{array}{l}\text { Gene } \\
\text { names }\end{array}$ & Diseases & & \\
\hline \multirow[t]{6}{*}{ Apoptosis } & 8 & CYCS; & Huntington's disease & DNA damage & Alzheimer's disease \\
\hline & & TNFRSF10D; & Thrombocytopenia & Bipolar & Rheumatoid arthritis \\
\hline & & $\begin{array}{l}\text { CASP7; } \\
\text { MAP3K1; }\end{array}$ & Diabetes mellitus type I & Renal cell carcinoma & Myeloproliferative disorders \\
\hline & & PRKCZ; MPL; & & & \\
\hline & & TNFRSF19 & Leukemia & Diabetes mellitus type ॥ & Amyotrophic lateral Sclerosis \\
\hline & & & Pancreatitis & Carcinoma (multiple types) & Multiple sclerosis \\
\hline \multirow[t]{8}{*}{ Cell cycle } & 20 & $\begin{array}{l}\text { BRCA2; } \\
\text { POLD2; }\end{array}$ & Spontaneous abortion & Genomic instability & Drug toxicity \\
\hline & & $\begin{array}{l}\text { CDC14A; } \\
\text { CDT1; }\end{array}$ & & & \\
\hline & & $\begin{array}{l}\text { CHAFIA; } \\
\text { WRN; }\end{array}$ & Endometriosis & Anemia & Li-Fraumeni Syndrome \\
\hline & & $\begin{array}{l}\text { IPO5; KIF23; } \\
\text { TTK; }\end{array}$ & Aneuploidy & Amyotrophic lateral Sclerosis & Diabetes mellitus type I \\
\hline & & $\begin{array}{l}\text { MSH2; } \\
\text { CASC5; }\end{array}$ & & & \\
\hline & & $\begin{array}{l}\text { PRKCZ; } \\
\text { CASPT; }\end{array}$ & Carcinoma (multiple types) & Diabetes mellitus type ॥ & Werner Syndrome \\
\hline & & SERPINB13; & & & \\
\hline & & LATS1 & Male infertility & Rheumatoid arthritis & osteoporosis \\
\hline \multirow[t]{4}{*}{ Development } & 11 & $\begin{array}{l}\text { NCOA6; } \\
\text { PABPN1; }\end{array}$ & Carcinoma (multiple types) & Endometriosis & Asthma \\
\hline & & LTF; MAP3K1; & & & \\
\hline & & $\begin{array}{l}\text { TLL1; YAP1; } \\
\text { SPEN; }\end{array}$ & Deglutition disorders & Male infertility & Coronary disease \\
\hline & & IL18R1 & $\begin{array}{l}\text { Oculopharyngeal Muscular } \\
\text { dystrophy }\end{array}$ & Li-Fraumeni Syndrome & Idiopathic pulmonary fibrosis \\
\hline \multirow[t]{3}{*}{ DNA repair } & 9 & $\begin{array}{l}\text { BRCA2; } \\
\text { CHAFIA; }\end{array}$ & Genomic instability & Werner Syndrome & Fanconi anemia \\
\hline & & $\begin{array}{l}\text { FANCM; } \\
\text { RAD51L1; }\end{array}$ & Carcinoma (multiple types) & Diabetes mellitus type I & Drug toxicity \\
\hline & & WRN; MSH2 & & & \\
\hline \multirow[t]{11}{*}{ Inflammation } & 22 & CYCS; SOCS6; & Huntington's disease & $\begin{array}{l}\text { Severe combined } \\
\text { Immunodeficiency }\end{array}$ & Stomach ulcer \\
\hline & & $\begin{array}{l}\text { BDKRB 1; } \\
\text { HPRT1; }\end{array}$ & & & \\
\hline & & IL18R1; IL7R; & Thrombocytopenia & Multiple sclerosis & Ataxia \\
\hline & & $\begin{array}{l}\text { MAP3K1; } \\
\text { 1L27RA; }\end{array}$ & Asthma & Rheumatoid arthritis & Leukemia \\
\hline & & PRKCZ; OAS2; & Diabetes mellitus type I & Myeloproliferative disorders & Sarcoidosis \\
\hline & & $\begin{array}{l}\text { CD53; } \\
\text { LAMA3; }\end{array}$ & & & \\
\hline & & $\begin{array}{l}\text { LAMA4; } \\
\text { IL174A; }\end{array}$ & Diabetic nephropathies & Crohn's disease & Carcinoma (multiple types) \\
\hline & & HABP2; KLKB1 & & & \\
\hline & & & $\begin{array}{l}\text { Autosomal dominant } \\
\text { polycystic kidney }\end{array}$ & Sjogren's syndrome & Gout \\
\hline & & & Rhinitis & Nasal polyps & Telangiectasis \\
\hline & & & Lesch-Nyhan syndrome & Arteriosclerosis & Genome instability \\
\hline \multirow[t]{2}{*}{ Metabolism } & 4 & P2RX2; LTF; & Anoxia & Encephalitis & Rheumatoid arthritis \\
\hline & & $\begin{array}{l}\text { ACACB; } \\
\text { PRKCZ }\end{array}$ & Carcinoma (multiple types) & Diabetes mellitus type ॥ & Glomberulonephritis \\
\hline $\begin{array}{l}\text { Nervous } \\
\text { System }\end{array}$ & 17 & CDK5RAP2; & Microcephaly & Lipodystrophy & Rheumatoid arthritis \\
\hline
\end{tabular}


Table 2 Diseases associated with genes containing nsSNPs (Continued)

\begin{tabular}{|c|c|c|c|c|c|}
\hline & & $P 2 R X 2$ & Anoxia & Mucolipidoses & Fragile X Syndrome \\
\hline & & $\begin{array}{l}\text { ST8S1A2; } \\
\text { HTR1F; }\end{array}$ & Schizophrenia & Pain & Liver cirrhosis \\
\hline & & $\begin{array}{l}\text { SCN10A; } \\
\text { SLC28A2; }\end{array}$ & Parkinson's disease & Asthma & Diabetes mellitus type I \\
\hline & & $\begin{array}{l}\text { MCOLN1; } \\
\text { DLGAP4; }\end{array}$ & Glioma & Acquired immunodeficiency & \\
\hline & & $\begin{array}{l}\text { SYT8; } \\
\text { ATP2B3; }\end{array}$ & & & \\
\hline & & $\begin{array}{l}\text { MAPIA; } \\
\text { LAMA4; }\end{array}$ & & & \\
\hline & & $\begin{array}{l}\text { MAP3K1; } \\
\text { AHNAK; }\end{array}$ & & & \\
\hline & & AFF2; CASP7 & & & \\
\hline $\begin{array}{l}\text { Protein } \\
\text { folding }\end{array}$ & 4 & $\begin{array}{l}\text { CHAF1A; } \\
\text { GRPELI; }\end{array}$ & Aneurysm & Pulmonary fibrosis & Diabetes mellitus type I \\
\hline & & $\begin{array}{l}\text { HSPA4; } \\
\text { EEFIA2; }\end{array}$ & & & \\
\hline & & & Carcinoma (multiple types) & Graft vs. host disease & Paralysis \\
\hline Reproduction & 13 & $\begin{array}{l}\text { SPDY1; } \\
\text { CENPI; }\end{array}$ & Gastrointestinal diseases & Hyperaldosteronism & Genomic instability \\
\hline & & $\begin{array}{l}\text { BRCA2; } \\
\text { NCOA4; }\end{array}$ & & & \\
\hline & & RBAK; CASC5; & Carcinoma (multiple types) & Diabetes mellitus type ॥ & Oligospermia \\
\hline & & $\begin{array}{l}\text { PRKCZ; } \\
\text { MAP3K1 }\end{array}$ & & & \\
\hline & & & anemia & & \\
\hline $\begin{array}{l}\text { Signal } \\
\text { transduction }\end{array}$ & 19 & $\begin{array}{l}\text { BRCA2; } \\
\text { CASP7; }\end{array}$ & Spontaneous abortion & Arteriosclerosis & Dilated cardiomyopathy \\
\hline & & $N C O A 6 ; l L 7 R$ & & & \\
\hline & & CYCS; BAMBI; & Carcinoma (multiple types) & Hypertension & Asthma \\
\hline & & $\begin{array}{l}\text { KLKB1; } \\
\text { IL17RA; }\end{array}$ & & & \\
\hline & & $\begin{array}{l}\text { MAP3K1; } \\
\text { DSG2; }\end{array}$ & Huntington's disease & $\begin{array}{l}\text { Severe combined } \\
\text { immunodeficiency }\end{array}$ & Sarcoidosis \\
\hline & & PRKCZ; IFI44; & & & \\
\hline & & $\begin{array}{l}\text { IL18R1; } \\
\text { HABP2; }\end{array}$ & Thrombocytopenia & Multiple sclerosis & Diabetes mellitus type I \\
\hline & & & Rheumatoid arthritis & Alzheimer's disease & $\begin{array}{l}\text { Arrhythmogenic right } \\
\text { ventricular dysplasia }\end{array}$ \\
\hline Transcription & 8 & $\begin{array}{l}\text { NCOA6; } \\
\text { BRCA2; }\end{array}$ & Retinoblastoma & $\begin{array}{l}\text { Oculopharyngeal muscular } \\
\text { dystrophies }\end{array}$ & Rheumatoid arthritis \\
\hline & & $\begin{array}{l}\text { MAP3K1; } \\
\text { TAF3; }\end{array}$ & & & \\
\hline & & PABPN1 & Spontaneous abortion & Aneuploidy & Deglutition disorders \\
\hline & & & Carcinoma (multiple types) & Infertility & \\
\hline Transport & 11 & KCNH3; LTF; & Anemia & Genomic instability & Liver cirrhosis \\
\hline & & $\begin{array}{l}\text { MSH2; } \\
\text { NCOA4; }\end{array}$ & Alzheimer's disease & Crohn's disease & Obesity \\
\hline & & PPARGC1B; & Epilepsy & Diabetes mellitus type ॥ & Barrett esophagus \\
\hline & & ZNF217; & Carcinoma (multiple types) & Gastritis & progeria \\
\hline & & $\begin{array}{l}\text { ME2;P2RX2; } \\
\text { ANK3; }\end{array}$ & & & \\
\hline & & $\begin{array}{l}\text { LATS1; } \\
\text { MAT2A }\end{array}$ & & & \\
\hline
\end{tabular}

A list of genes containing probably or possibly damaging nsSNPs as predicted by PolyPhen-2 was submitted for network analysis in GeneGo. Characterization of the genes containing nsSNPs is achieved by listing the general cellular process (column 1), the total number of deleterious nsSNPs in the genes that GeneGo annotated (column 2), the genes associated with the cellular process (column 3), and the specific diseases that have been associated by GeneGo with the genes in question (columns 4-6). 
and the two genomes have diverged more than $6 \%$ in overall DNA sequence since that time [23]. Consequently, except in unusual loci such as HLA, homologous basepair positions that are polymorphic SNPs in both species are highly unlikely to represent shared ancestral polymorphism that has been retained for more than 20 million years in both lineages. Rather, most shared SNP positions will reflect parallel mutational events that created variation at the same site in both species. We converted all 3,038,166 validated rhesus SNPs from this study to hg19 human coordinates, and $2,775,850$ of those SNPs successfully converted using the Galaxy hosted UCSC liftOver tool. Of these converted SNPs, 90,086 exactly matched the location of human SNPs in dbSNP Build 132, representing a 3.2\% overlap. Given that there are 29,100,846 human SNPs (chromosomes 1-22, X) in build 132, about 1\% of the human genome, if the specific locations of SNPs in the human and rhesus genomes were entirely uncorrelated then we would only expect about one-third as many polymorphic basepair positions to be shared in the two species. This correlation between the locations of SNPs in rhesus monkeys and humans is almost certainly influenced in part by constraints on which sites within each genome can tolerate polymorphism. But other mechanisms, such as correlations in mutation rates at homologous sites across primate genomes, may also be involved.

\section{Discussion}

In this study, we validated 3,038,166 SNPs and identified an additional 11,382,666 potential SNPs for the Indianorigin rhesus macaque. Both Chinese and Indian-origin rhesus macaques are used in biomedical research, but Indian-origin animals are more prevalent. Furthermore, the rhesus macaque reference genome was produced using an individual of Indian-origin [23]. At the time we began this study, very few SNPs or other variants were confirmed or validated in the rhesus genome. By taking advantage of the falling cost of next-gen resequencing and utilizing all of the existing sequence data available, we were able to validate a large genome-wide series of SNPs. Different sequencing technologies exhibit distinct probabilities of different sequencing errors that will increase false positive rates in calling novel SNPs [38]. SNPs validated in a single individual using multiple sequencing technologies avoid this problem, thus increasing confidence in the SNP calls common to the two data sets. In addition to inherent sequencing chemistry concerns, low coverage sequencing can suffer from read coverage limitations, creating false negative data because the SNP calling software will discard significant proportions of legitimate SNPs due to low coverage (Figure 4). For this reason, we chose to utilize reduced

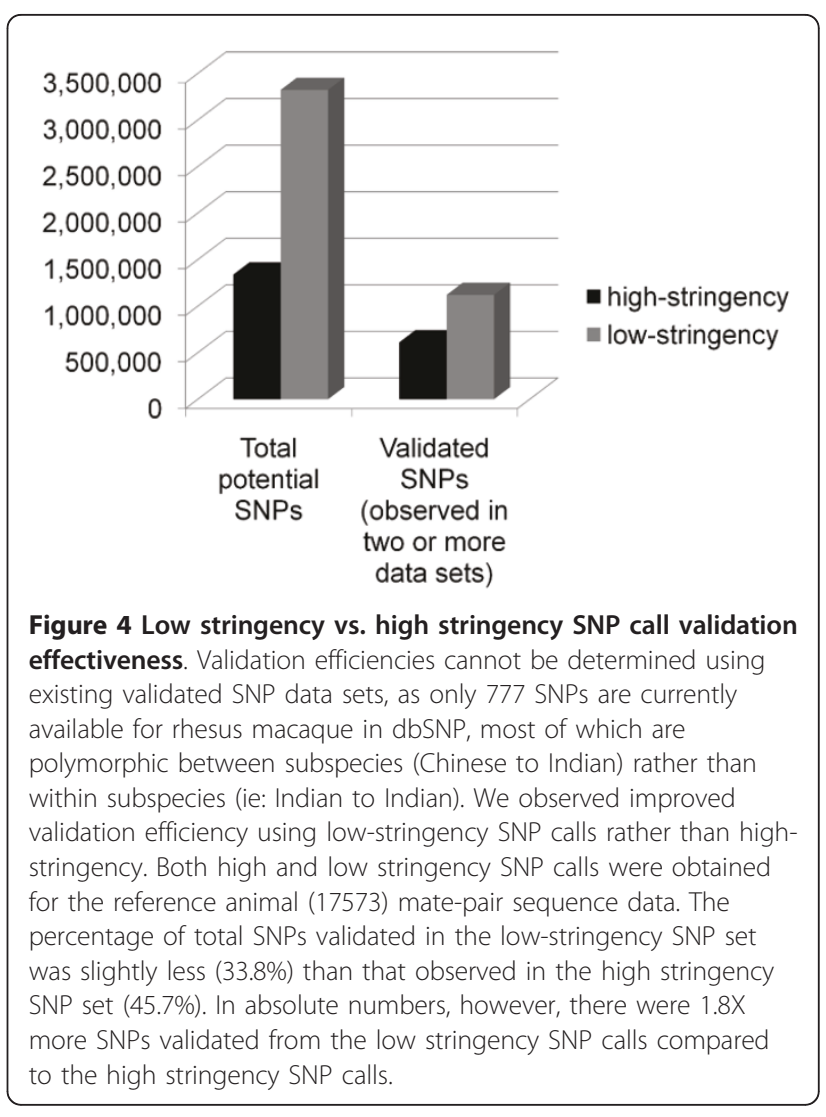

stringency SNP calls from corona_lite and AtlasSNP2. Our methodology minimizes both false positive and false negative SNP calls by using reduced-stringency SNP calling standards but requiring that each SNP location and both alleles be confirmed in data obtained from different animals and/or different chemistries. In addition, most of our comparisons utilized comparisons of SNP data sets that were called by different algorithms. Studies of human population variation from the 1000 Genomes Project [39] have shown that utilizing the intersection of different SNP calling methods to hone in on real SNP data reduces the false positive SNP error rate by $30-50 \%$. This validated SNP list represents a substantial increase in the number of known rhesus SNPs, and therefore is an important resource for research involving this species. These data will facilitate the discovery of functional SNPs, the development of new disease models, and genetic linkage studies, as well as providing a valuable resource for colony management.

The most interesting SNPs in any data set are those that directly alter biological function, but these are also the most challenging to identify. While functional SNPs can be in coding (non-synonymous or splice site SNPs) or non-coding regions (non-coding RNAs, regulatory regions), the simplest SNPs to evaluate for function are 
in coding regions. We took advantage of the close evolutionary relationship between rhesus macaques and humans to generate hypotheses about the functionality of the non-synonymous SNPs we identified. With 411 SNPs that are predicted to be probably damaging by PolyPhen-2 [36], we have begun extending the information concerning potentially significant functional variation in this species. Out of these SNPs, several occur in genes associated with behavior (Scn10A), metabolism (Ppgca1b), cancer (Brca2), and immune response (Map3k1, OAS2, IL18R1).

Our study has identified substantial genetic variation among Indian-origin rhesus monkeys. Previous information [24] shows that separate SNP identification efforts are required in Chinese-origin and Indian-origin monkeys. Only about one-third of SNPs that are polymorphic in one population are also polymorphic in the other [27]. Thus, the information we report here is potentially helpful to investigators using Chinese-origin rhesus macaques, but additional work to discover SNPs in that population is warranted, just as information concerning variability among Chinese-origin animals is only partially relevant to our study population.

While this study produced greater than 3,000 fold more SNPs than had previously been known in rhesus macaques (both Indian and Chinese), it is not exhaustive. Roughly $25 \%$ of each potential SNP list was validated using our methodology (Figure S1, Additional file 1). Further validation of the remaining potential SNPs may proceed either through additional sequencing of the large data sets, or by sequencing additional animals. Currently, there are more than 37,821,067 human SNPs in dbSNP. Not only does that predict a lower bound of $\sim 34$ million rhesus macaque SNPs yet to be identified, but the hypothesized increased genetic variation in rhesus macaques indicates that we may identify many more SNPs than have been validated in humans. This study focused upon the genome-wide identification of SNPs in an effort to meet a wide variety of needs in the primate research community. Future work highlighting coding variants could take advantage of deep re-sequencing of exomes or re-sequencing specific loci where prior information suggests a potential role in disease or phenotype.

\section{Conclusions}

Our study identified more than 14 million potential single nucleotide variants and validated $\sim 3$ million of these variants. Various strategies can be used to detect and validate SNPs in any given species. We present an approach that combines different types of next-gen sequence data with low stringency bioinformatic filters, and relies upon differences among individuals and across sequencing chemistries to validate variants. Validated SNP coverage was relatively even throughout the genome. Additionally, a proportion of validated SNPs are predicted to be probably damaging in rhesus homologues of genes that are known to contribute to disease in humans. This increase in information concerning genetic variation in Indian-origin rhesus macaques opens up many new opportunities for the research community in evolutionary studies, the use of rhesus macaques as models of human disease, and studies of primate diversity.

\section{Materials and methods Ethics Statement}

All relevant animal procedures in this project were approved by the appropriate Institutional Animal Care and Use committees from the Southwest National Primate Research Center or the University of Wisconsin, and comply with the federal regulations. Genomic DNA for sequencing was obtained either from frozen tissues collected previously as part of other research projects or from blood samples collected from live animals by venupuncture, using appropriate anesthetics.

\section{Data sources}

We began our analysis with the Sanger sequence data generated as part of the original rhesus macaque genome project [23]. A single animal (animal ID 17573) from the Southwest National Primate Research Center (San Antonio, TX) Indian-origin rhesus colony was sequenced to approximately $5.2 \mathrm{X}$ coverage $(18.4 \mathrm{~Gb}$ of raw sequence) using Sanger whole genome shotgun methods. Additional details of that reference genome analysis have been published previously [23]. From this Sanger data, approximately 4.3 million basepair positions were called as potentially heterozygous using SNPdetector [40] (Houston, TX). We obtained additional genomic DNA from animal 17573 and created two libraries for SOLiD sequencing, one library for fragment sequencing and one for mate-pair sequencing (Table S1, Additional file 3). Using published methods [41,42] (50bp fragment (SRA accession: SRX029055) and $25 \times 25$ bp mate pair data (SRA accession: SRX029056)) we generated $5 \mathrm{X}$ fragment sequence coverage and approximately $8 \mathrm{X}$ mate-pair sequence coverage. The proportion of matching beads to total beads was: $49.52 \%$ for the fragment library and $55.22 \%$ for the mate pair library (F3+R3). Following the re-sequencing of the original reference animal, we selected two unrelated Indian-origin rhesus macaques (animal ID's r1766 (SRA accession: SRX029057) and r02120 (SRA accession: SRX029058)) from the population at the Wisconsin National Primate Research Center (University of Wisconsin, Madison). Each DNA sample was used to produce fragment sequencing libraries, and each library sequenced to low coverage (50bp HiDRA, r1766 to 
$5.8 \mathrm{X}, \mathrm{r} 02120$ to $3.3 \mathrm{X})$. The ratio of matching beads to total beads was $58.64 \%$ and $49.89 \%$, respectively. The library for r02120 exhibited a decreased degree of unique beads relative to $\mathrm{r} 1766$ and 17573 , but did produce a substantial amount of high-quality data.

We also downloaded additional 454 sequence reads from NCBI (26.2 Mb) produced during the original rhesus sequencing project [23] from a comparison of rhesus macaque sub-species (8 Chinese-origin and 8 Indian-origin unrelated rhesus macaques). SNPs were called using these reads and the default settings for AtlasSNP2 [43] without filtering for coverage. Sub-species comparison read data was provided at $0.5 \mathrm{X}$ coverage [23]. 142,781 potential SNPs were identified in the sub-species comparison data set.

Several lists of SNP calls were generated previously (Table 3). MamuSNP provided a list of 22,892 potential SNPs [25,26](http://mamusnp.ucdavis.edu/query.php[44]). ENCODE consisted of a list of 1,672 validated SNPs out of which 1,467 have been previously described [24]. Additional validated SNPs were downloaded from dbSNP (http://www.ncbi.nlm.nih.gov/projects/SNP/[16]) and MonkeySNP (http://monkeysnp.ohsu.edu/snp/[45]), for a total of 765 SNPs at the time of download. Overlap was almost exact (763 of 765) between these two data sources and the data set will hereafter be referred to as dbSNP.

\section{SNP calling and validation methods}

Identification of specific bases that are heterozygous in a given individual was accomplished using different methods for different sequencing technologies. The original Sanger read data for the reference animal was searched for SNPs (i.e. heterozygous basepair positions) using SNPdetector [40]. For the SOLiD read data, corona_lite v4.0 r2.0 (Life Technologies, Carlsbad, CA) was used. SNPs were called in the sub-species comparison data using AtlasSNP2 [43] as described above.
We considered all basepair positions called as heterozygous in any one dataset as "potential SNPs." In order to classify a given SNP as "validated," we required the specific base pair position be called as an identical heterozygote in two independent datasets. This could mean either observing that position as heterozygous in the same individual using two different sequencing methods, or observing that position to be heterozygous, with identical alleles, in two different animals. Two complimentary methods were used to identify SNPs in the data sets described above: positional-allele comparison and e-genotyping.

Positional-allele comparison relies upon parameters of sequence quality and read coverage as implemented in various SNP calling programs (in this study we used SNPdetector, corona_lite, and AtlasSNP2). This method compares lists of identified potential SNPs by chromosome, base pair location, and both alleles. The comparison method can identify multiple SNPs clustered close together or those near to breakpoints in the reference sequence. But this method has a potentially higher false negative rate compared with e-genotyping, due to the relative stringency of the SNP calling programs used upstream. To reduce false negative calls, we used stringency thresholds somewhat lower than those used in some other studies. Our settings generally retained potential SNPs with read quality scores equivalent to a Phred score of 20 and a minimum of 2 reads covering each variant allele call.

E-genotyping is a novel approach that takes advantage of an a priori defined set of potential SNPs, and then tests raw reads (color-space or sequence space data) directly for exact probe matches to the previously defined SNP location and sequence. A probe region consists of 15 bases on each side of the potential SNP. These flanking regions must be pre-defined, and must match the target reads in the new sequence dataset exactly, with different reads providing exact matches to

Table 3 Data sources

\begin{tabular}{|c|c|c|c|c|}
\hline Chemistry & Name & DNA source & $\begin{array}{l}\text { SNP calling } \\
\text { software }\end{array}$ & References \\
\hline Sanger & Assembly & Animal 17573 \& & SNPdetector & Gibbs, et al. (2007) [23], Wheeler (pers. comm.) \\
\hline Sanger & ENCODE & 47 pooled individuals & SNPdetector & Hernandez, et al. (2007) [24] \\
\hline SOLiD & 17573 fragment & Animal 17573 \& & Corona_lite & \\
\hline$\overline{S O L i D}$ & 17573 mate-pair & Animal 17573 ᄋ & Corona_lite & \\
\hline SOLiD & $r 1766$ & Animal r1766 ᄋ & Corona_lite & \\
\hline SOLiD & r02120 & Animal r02120 ơ & Corona_lite & \\
\hline 454 & $\begin{array}{l}\text { Sub-species } \\
\text { comparison }\end{array}$ & 16 pooled individuals & AtlasSNP & Gibbs, et al. (2007) [23] \\
\hline 454 & MamuSNP & $\begin{array}{l}\sim 7 \text { pooled } \\
\text { individuals }\end{array}$ & Unknown & Malhi, et al. (2007) [25], http://mamusnp.ucdavis.edu/query.php[44] \\
\hline unknown & dbSNP/MonkeySNP & $\begin{array}{l}\text { Unknown, }>30 \\
\text { estimated }\end{array}$ & Unknown & $\begin{array}{l}\text { http://monkeysnp.ohsu.edu/snp/[45], http://www.ncbi.nlm.nih.gov/ } \\
\text { projects/SNP/[16] }\end{array}$ \\
\hline
\end{tabular}


the two expected alleles in the potential SNP position. This novel method of validating potential SNPs in a new read set is restricted by the exact matching parameter that confers the high specificity. When e-genotype was used to screen human 1000 Genomes read data for known SNPs (from dbSNP) in the same individual, the error call rates were extremely low for miscalled homozygotes (called as wrong homozygote $(0.002 \%)$ ) and heterozygotes (called as wrong heterozygote $(1.01 \%)$ or homozygote (1.52\%)) (Figure S3, Additional file 4). dbSNP concordance in human data from the 1000 Genomes pilot study [39] using e-genotype indicates that the total miscall rate is $\sim 2.6 \%$ (Figure S3, Additional file 4). When extremely high coverage or low coverage SNPs (delineated by dotted lines on figure) are removed from consideration (high coverage SNPs are typically non-specific and low coverage SNPs are often miscalled hets where coverage was simply insufficient to detect both alleles), the miscall rate is calculated to be $1.6 \%$. Because of the stringency of probe placement, e-genotype has much reduced effective read coverage for any SNP, but of the SNPs that are called by e-genotype, the probability of false positive calls is extremely low. E-genotype has significantly reduced power to detect clustered SNPs, especially in linkage disequilibrium, when they fall within the 31 base pair probe region. E-genotype is unable to identify SNPs located within 15 base pairs of gap regions in the reference sequence or chromosome ends.

We developed a perl pipeline to compare chromosome, base pair locations, and both alleles for all pairwise comparisons of SNP lists. At least one read of sufficient quality (equivalent to a minimum Phred score of 20) for each allele in each data set was required for a positive result, and all instances with more than 200 reads covering an allele were removed irrespective of average read coverage for the data set, due to presumed mis-mapping or non-unique mapping. Once these comparisons were completed, all of the validated SNPs were appended in a single file and duplicates were removed by chromosome/base-pair determination.

E-genotyping (http://is04607.com/ drio/egenotype/ [29]) was performed on all the SOLiD read sets from animals 17573, r1766 and r02120. The probe sets were created from: a) the Sanger data from the original reference animal (17573), b) the novel SNP calls from corona_lite for $\mathrm{r} 1766$ and $\mathrm{r} 02120$, or c) the reads produced using Roche 454 methods on unrelated animals in the original rhesus genome paper [23]. All the probe sets were checked for unique mapping locations by mapping to the reference genome assembly prior to running egenotype on experimental read sets. E-genotyping results with at least one read for each allele were considered to be positive when comparing data from different chemistries.
We annotated the SNPs using a Java tool that accessed Ensembl (build 57, March 2010). Annotation of SNPs included the genomic placement (intronic, intergenic, synonymous coding, nonsynonymous coding, 5'UTR, 3'UTR, or splice site) for all applicable transcripts. If a SNP was determined to fall in a genic region (coding and non-coding), the following annotations were added as applicable: gene name, gene function, codon, reference amino acid, variant amino acid, protein position.

All of the validated SNPs have been submitted to dbSNP (http://www.ncbi.nlm.nih.gov/projects/SNP/[16]) (ss numbers available in Table S2, Additional files 5, 6, 7, 8, 9 and 10). Unvalidated potential SNPs will be available at our lab Genboree site (http://genboree.org/javabin/project.jsp?projectName=Rhesus\%20SNPs\%20using\% 20Next-Gen\%20Sequencing\&isPublic=Yes) [30].

\section{Polyphen-2 analysis and GeneGo analysis}

Non-synonymous SNPs (nsSNPs) from this validated list of rhesus macaque SNPs were converted to human genome (hg18) co-ordinates using the UCSC liftOver tool from Genboree Galaxy and then analyzed for possible functional significance using PolyPhen-2 (http://genetics. bwh.harvard.edu/pph2/[35]) [36]. All SNPs that did not convert cleanly to unique locations in hg18, or where the human reference allele at that location matched the rhesus variant allele, were removed from further analysis. The resulting list of human homologues of our rhesus nsSNPs was submitted as a batch file for HumDiv model analysis in PolyPhen-2. Polyphen-2 utilizes both homologous sequence alignment as well as known protein 3D crystal structures to predict the potential effect of any given polymorphism upon protein function. Polymorphisms are tested for the potential damaging effect upon protein function by normalization to known human deleterious alleles in UniProt and use of a naïve Bayes classifier. All genes containing SNPs that were classified as damaging (probably or possibly) in the PolyPhen-2 output were submitted to GeneGo (St. Joseph, MI, http://www.genego.com[37]) for network analysis. Each gene was associated with a generalized function and proposed disease network.

\section{Additional material}

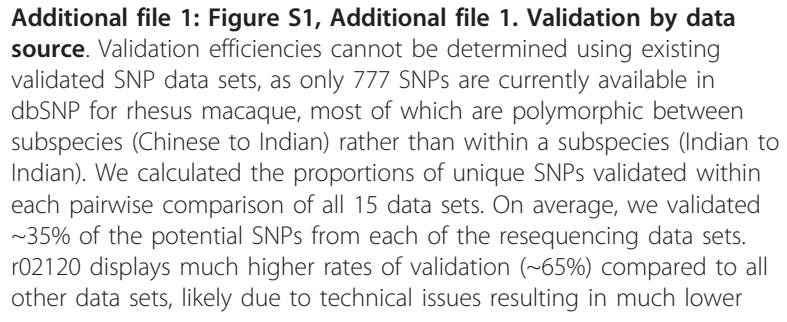


coverage. The Sub-species comparison data set exhibited similar validation rates $(\sim 40 \%)$ to the resequenced data sets, which may be explained by the fact that this data set contained many more SNPs than the other non-resequenced data sets. The smaller data sets (MamuSNP, ENCODE, dbSNP, and all e-genotyping data sets) all displayed significantly lower validation rates $(\sim 10 \%)$ due both to these data sets being generated using both Chinese and Indian origin animals rather than only Indian animals and to the small number of SNPs in each of these sets.

Additional file 2: Figure S2, Additional file 2. Sanger read coverage for validated SNPs. All SNPS that were found in common between r1766, r02120, and 17573 (306,782, Figure 1B) were tested for Sanger read coverage using the 17573 Sanger data by looking for the same location and allele SNP call in the Sanger output (red bars). Average genome-wide Sanger read coverage was 5.2X. We analyzed an identical number of randomly selected SNPs that were detected in only two of the three resequenced animals (blue bars) to test if there were differences in the distribution of read coverage for the two SNP data sets. The distributions are indistinguishable, however, and the proportions of tested SNPS exhibiting 11+ read coverage are not statistically different (3.8\% for SNPs in all three animals, 3.6\% for SNPs detected in only two animals).

Additional file 3: Table S1, Additional file 3. SOLiD sequencing statistics. When using our new SOLiD data or prior data from the literature, our validation procedure only validated two alleles for any location, regardless of the data sets being compared. However, for some SNPs, neither of the two validated alleles matched the published reference allele. Some (8) of these are likely due to Indian-origin vs. Chinese-origin differences. For the vast majority, however, there are two possibilities, each of which explains an undefinable proportion of these SNPS: (1) a proportion of the reference allele (Sanger) calls are wrong, and (2) a small proportion of the apparent three allele SNPs represent true SNPs with three valid alleles in Indian-origin rhesus macaques.

Additional file 4: Figure S3, Additional file 4. dbSNP concordance of e-genotype. One human sample of SOLiD data (26X coverage) from the 1000 Genomes pilot data was analyzed for the $\sim 1$ million SNPs identified in the project for that sample. Color coding for the bars as well as the lines is as follows: good homozygous calls (blue), heterozygotes called as homozygotes (yellow), homozygotes called as the wrong homozygote (grey), good heterozygote calls (green), and erroneously called heterozygotes (red). The total miscall rate was $\sim 2.6 \%$. Excluding very high and very low coverage errors (shown outside of dotted lines, due to bad heterozygous SNP calls from repetitive regions or coverage too low to detect heterozygotes, respectively) the miscall rate was determined to be $1.6 \%$. Overall probe coverage was reduced to $8.2 X$ due to the extremely stringent requirement of exact matching of probes for the full $31 \mathrm{bp}$ probe length. Percentages of homozygous or heterozygous calls that fit into each category of good or error calls were linearly graphed relative to probe coverage, indicating that errors were much more likely at very high and very low probe coverage, while good calls were most likely in intermediate coverage ranges.

Additional file 5: Table S2, Additional file 5. Part 1 of dbSNP ss numbers for validated rhesus macaque SNPs. The first part of six of a table listing of all of the ss numbers from submission of the validated rhesus macaque SNPs to dbSNP. The ss numbers are not contiguous throughout the full set of 3,038,166 SNPs.

Additional file 6: Table S2, Additional file 6. Part 2 of dbSNP ss numbers for validated rhesus macaque SNPs. The second part of six of a table listing of all of the ss numbers from submission of the validated rhesus macaque SNPs to dbSNP. The ss numbers are not contiguous throughout the full set of 3,038,166 SNPs.

Additional file 7: Table S2, Additional file 7. Part 3 of dbSNP ss numbers for validated rhesus macaque SNPs. The third part of six of a table listing of all of the ss numbers from submission of the validated rhesus macaque SNPs to dbSNP. The ss numbers are not contiguous throughout the full set of 3,038,166 SNPs.

Additional file 8: Table S2, Additional file 8. Part 4 of dbSNP ss numbers for validated rhesus macaque SNPs. The fourth part of six of a table listing of all of the ss numbers from submission of the validated rhesus macaque SNPs to dbSNP. The ss numbers are not contiguous throughout the full set of 3,038,166 SNPs.

Additional file 9: Table S2, Additional file 9. Part 5 of dbSNP ss numbers for validated rhesus macaque SNPs. The fifth part of six of a table listing of all of the ss numbers from submission of the validated rhesus macaque SNPs to dbSNP. The ss numbers are not contiguous throughout the full set of 3,038,166 SNPs.

Additional file 10: Table S2, Additional file 10. Part 6 of dbSNP ss numbers for validated rhesus macaque SNPs. The sixth part of six of a table listing of all of the ss numbers from submission of the validated rhesus macaque SNPs to dbSNP. The ss numbers are not contiguous throughout the full set of $3,038,166$ SNPs.

\section{Acknowledgements}

We thank Snekalatha Raveendran for data and file management, and the Wisconsin National Primate Research Center for blood samples from the two non-reference study animals. The reference animal (17573) blood sample was provided by the Southwest National Primate Research Center. Matthew Bainbridge provided a java tool that facilitated SNP annotation from Ensembl.

Funding Statement

This work was supported by the NIH grant 2 U54 HG003273 to the Human Genome Sequencing Center (RG), NCRR grant NIH-R24-RR15383 (JR), and internal funding from Baylor College of Medicine (JR). Genboree system development has been supported by the $\mathrm{NIH}$ grants U01 DA025956 and R01 HG004009 (AM). Colony management was facilitated by the NIH grants 5RO1-MH081884 (NK), 5RO1-MH046729 (NK), and 5P50-MH84051 (NK, PI: Richie Davidson).

\section{Author details}

'Human Genome Sequencing Center, Department of Molecular and Human Genetics, Baylor College of Medicine, Houston, Texas 77030, USA. 2Department of Molecular and Human Genetics, Baylor College of Medicine, Houston, Texas 77030, USA. ${ }^{3}$ Southwest National Primate Research Center, San Antonio, Texas 78245, USA. ${ }^{4}$ Department of Psychiatry, the HealthEmotions Research Institution, University of Wisconsin-Madison, Madison, Wisconsin, 53719, USA. ${ }^{5}$ Department of Psychology, Waisman Laboratory for Brain Imaging and Behavior, University of Wisconsin-Madison, Madison, Wisconsin, 53719, USA.

\section{Authors' contributions}

$J R, G L F$ and RG contributed to project conception. GLF, FY, JGR, DMM, AM, DAW, KCW, RG and JR contributed to experimental design and approach. Software tools were created by: GLF (perl comparison tool pipeline), DRD and JGR (e-genotype), FY (AtlasSNP), RAH and AM (Genboree), YR (SNPdetector). Experiments were performed by GLF, MR, DRD, DC, and RAH. Data was analyzed by: GLF, MR, DRD, DC, YR. Resources to generate data were provided by: DMM and RG (library and sequencing resources), YR and DAW (initial list of reference animal heterozygotes), SS and NHK (rhesus DNA), JR (rhesus DNA). The manuscript was prepared by GLF and JR. All authors read and approved the final manuscript.

\section{Competing interests}

The authors declare that they have no competing interests.

Received: 7 January 2011 Accepted: 13 June 2011

Published: 13 June 2011

\section{References}

1. Capitanio JP: Individual differences in emotionality: social temperament and health. Am J Primatol 2010.

2. Kinnally EL, Capitanio JP, Leibel R, Deng L, LeDuc C, Haghighi F, Mann JJ: Epigenetic regulation of serotonin transporter expression and behavior in infant rhesus macaques. Genes Brain Behav 2010, 9(6):575-582.

3. Barr CS, Newman TK, Becker ML, Parker CC, Champoux M, Lesch KP, Goldman D, Suomi SJ, Higley JD: The utility of the non-human primate: 
model for studying gene by environment interactions in behavioral research. Genes Brain Behav 2003, 2(6):336-340.

4. Kalin NH, Shelton SE: Nonhuman primate models to study anxiety, emotion regulation and psychopathology. Ann N Y Acad Sci 2003, 1008:189-200

5. Oler JA, Fox AS, Shelton SE, Rogers J, Dyer TD, Davidson RJ, Shelledy W, Oakes TR, Blangero J, Kalin NH: Amygdalar and hippocampal substrates of anxious temperament differ in their heritability. Nature 2010, 466(7308):864-868

6. Tokuyama Y, Reddy AP, Bethea CL: Neuroprotective actions of ovarian hormones without insult in the raphe region of rhesus macaques. Neuroscience 2008, 154(2):720-731.

7. Xu J, Bernuci MP, Lawson MS, Yeoman RR, Fisher TE, Zelinski-Wooten MB, Stouffer RL: Survival, growth, and maturation of secondary follicles from perpubertal, young and older adult rhesus monkeys during encapsulated three-dimensional (3D) culture: effects of gonadotropins and insulin. Reproduction 2010, 140:685-697.

8. Cruzen C, Colman RJ: Effects of caloric restriction on cardiovascular aging in non-human primates and humans. Clin Geriatr Med 2009, 25(4):733-743.

9. Colman RJ, Anderson RM, Johnson SC, Kastman EK, Kosmatka KJ, Beasley TM, Allison DB, Cruzen C, Simmons HA, Kemnitz JW, Weindruch R: Caloric restriction delays disease onset and mortality in rhesus monkeys. Science 2009, 325(5937):201-204.

10. Vivian JA, Higley JD, Linnoila M, Woods JH: Oral ethanol selfadministration in rhesus monkeys: behavioral and neurochemical correlates. Alcohol Clin Exp Res 1999, 23(8):1352-1361.

11. Barr CS, Goldman D: Non-human primate models of inheritance vulnerability to alcohol use disorders. Addict Biol 2006, 11(3-4):374-385

12. Blin G, Nury D, Stefanovic S, Neri T, Guillevic O, Brinon B, Bellamy V, RuckerMartin C, Barbry P, Bel A, Bruneval P, Cowan C, Pouly J, Mitalipov S, Gouadon E, Binder P, Hagege A, Desnos M, Renaud JF, Menache P, Puceat M: A purified population of multipotent cardiovascular progenitors derived from primate pluripotent stem cells engrafts in postmyocardial infarcted nonhuman primates. J Clin Invest 2010, 120(4)::1125-1139.

13. Kay DB, Marsiske M, Suomi SJ, Higley JD: Exploratory factor analysis of human infant temperament in the rhesus monkey. Infant Behav Dev 2010, 33(1):111-114.

14. Valentine $L E$, Watkins DI: Relevance of studying $T$ cell responses in SIVinfected rhesus macaques. Trends Microbiol 2008, 16(12):605-611.

15. Shedlock DJ, Silvestri G, Weiner DB: Monkeying around with HIV vaccines: using rhesus macaques to define 'gatekeepers' for clinical trials. Nature Reviews Immunology 2009, 9(10):717-728.

16. dbSNP. [http://www.ncbi.nlm.nih.gov/projects/SNP/].

17. Lee AS, Gutierrez-Arcelus M, Perry GH, Vallender EJ, Johnson WE, Miller GM, Korbel JO, Lee C: Analysis of copy number variation in the rhesus macaque genome identifies candidate loci for evolutionary and human disease studies. Hum Mol Genet 2008, 17(8):1127-1136.

18. Degenhardt JD, de Candia P, Chabot A, Schwartz S, Henderson L, Ling B, Hunter M, Jiang Z, Palermo RE, Katze M, et al: Copy number variation of CCL3-like genes affects rate of progression to simian-AIDS in Rhesus Macaques (Macaca mulatta). PLoS Genet 2009, 5(1):e1000346.

19. Francis PJ, Appukuttan B, Simmons E, Landauer N, Stoddard J, Hamon S, Ott J, Ferguson B, Klein M, Stout JT, Neuringer M: Rhesus monkeys and humans share common susceptibility genes for age-related macular disease. Hum Mol Genet 2008, 17(17):2673-2680.

20. Vallender EJ, Priddy CM, Hakim S, Yang H, Chen GL, Miller GM: Functional variation in the $3^{\prime}$ untranslated region of the serotonin transporter in human and rhesus macaque. Genes Brain Behav 2008, 7(6):690-697.

21. Barr CS, Newman TK, Shannon C, Parker C, Dvoskin RL, Becker ML, Schwandt M, Champoux M, Lesch KP, Goldman D, et al: Rearing condition and rh5-HTTLPR interact to influence limbic-hypothalamic-pituitary-adrenal axis response to stress in infant macaques. Biol Psychiatry 2004, 55(7):733-738.

22. Vallender EJ, Priddy CM, Chen GL, Miller GM: Human expression variation in the mu-opioid receptor is paralleled in rhesus macaque. Behav Genet 2008, 38(4):390-395.

23. Gibbs RA, Rogers J, Katze MG, Bumgarner R, Weinstock GM, Mardis ER, Remington KA, Strausberg RL, Venter JC, Wilson RK, et al: Evolutionary and biomedical insights from the rhesus macaque genome. Science 2007, 316(5822):222-234
24. Hernandez RD, Hubisz MJ, Wheeler DA, Smith DG, Ferguson B, Rogers J, Nazareth L, Indap A, Bourquin T, McPherson J, et al: Demographic histories and patterns of linkage disequilibrium in Chinese and Indian rhesus macaques. Science 2007, 316(5822):240-243.

25. Malhi RS, Sickler B, Lin D, Satkoski J, Tito RY, George D, Kanthaswamy S, Smith DG: MamuSNP: a resource for Rhesus Macaque (Macaca mulatta) genomics. PLOS ONE 2007, 2(5):e438.

26. Satkoski JA, Malhi R, Kanthaswamy S, Tito R, Malladi V, Smith D: Pyrosequencing as a method for SNP identification in the rhesus macaque (Macaca mulatta). BMC Genomics 2008, 9:256.

27. Ferguson B, Street $S L$, Wright $H$, Pearson C, Jia Y, Thompson SL, Allibone P, Dubay CJ, Spindel E, Norgren RB Jr: Single nucleotide polymorphisms (SNPs) distinguish Indian-origin and Chinese-origin rhesus macaques (Macaca mulatta). BMC Genomics 2007, 8:43.

28. Street SL, Kyes RC, Grant R, Ferguson B: Single nucleotide polymorphisms (SNPs) are highly conserved in rhesus (Macaca mulatta) and cynomolgus (Macaca fascicularis) macaques. BMC Genomics 2007, 8:480.

29. E-genotype. [http://is04607.com/ drio/egenotype/].

30. Rogers Lab: Indian-origin Rhesus SNPs. [http://genboree.org/java-bin/ project.jsp?projectName=Rhesus\%20SNPs\%20using\%20Next-Gen\% 20Sequencing\&isPublic=Yes].

31. She X, Jiang Z, Clark RA, Liu G, Cheng Z, Tuzun E, Church DM, Sutton G, Halpern AL, Eichler EE: Shotgun sequence assembly and recent segmental duplications within the human genome. Nature 2004, 431(7011):927-930

32. Wallace JC, Korth MJ, Paeper B, Proll SC, Thomas MJ, Magness CL, ladonato SP, Nelson C, Katze MG: High-density rhesus macaque oligonucleotide microarray design using early-stage hresus genome sequence information and human genome annotations. BMC Genomics 2007, 8:28.

33. Noriega NC, Kohama SG, Urbanski HF: Gene expression profiling in the rhesus macaque: Methodology, annotation and data interpretation. Methods 2007, 49:42-49.

34. Norgren RB Jr: Annotation of the rhesus macaque genome. 4th International Conference on Primate Genomics Seattle, WA: Washington National Primate Research Center; 2010, 14.

35. Polyphen-2. [http://genetics.bwh.harvard.edu/pph2/].

36. Adzhubei IA, Schmidt S, Peshkin L, Ramensky VE, Gerasimova A, Bork P, Kondrashov AS, Sunyaev SR: A method and server for predicting damaging missense mutations. Nature Methods 2010, 7(4):248-249.

37. GeneGo. [http://www.genego.com].

38. Metzker ML: Sequence technologies-the next generation. Nature Reviews Genetics 2010, 11:31-46.

39. 1000 Genomes Project Consortium, D RM, Abecasis GR, Altshuler DL, Auton A, Brooks LD, Durbin RM, Gibbs RA, Hurles ME, McVean GA: A map of human genome variation from population-scale sequencing. Nature 2010, 467(7319):1061-1073.

40. Zhang J, Wheeler DA, Yakub I, Wei S, Sood R, Rowe W, Liu PP, Gibbs RA, Buetow KH: SNPdetector: a software tool for sensitive and accurate SNP detection. PLoS Comput Biol 2005, 1(5):e53.

41. Lupski JR, Reid JG, Gonzaga-Jauregui C, Rio Deiros D, Chen DC, Nazareth L, Bainbridge $M$, Dinh $H$, Jing C, Wheeler DA, McGuire AL, Zhang F, Stankiewicz P, Halperin JJ, Yang C, Gehman C, Guo D, Irikat RK, Tom W, Fantin NJ, Muzny DM, Gibbs RA: Whole-genome sequencing in a patient with Charcot-Marie-Tooth neuropathy. N Eng J Med 2010, 362(13):1181-1191

42. Harismendy O, Ng PC, Strausberg RL, Wang X, Stockwell TB, Beeson KY, Schork NJ, Murray SS, Topol EJ, Levy S, Frazer KA: Evaluation of next generation sequencing platforms for population targeted sequencing studies. Genome Biology 2009, 10(3):R32.

43. Shen Y, Wan Z, Coarfa C, Drabek R, Chen L, Ostrowski EA, Liu Y, Weinstock GM, Wheeler DA, Gibbs RA, Yu F: A SNP discovery method to assess variant allele probability from next-generation resequencing data. Genome Res 2010, 20(2):273-280.

44. MamuSNP. [http://mamusnp.ucdavis.edu/query.php].

45. Khouangsathiene S, Pearson C, Street S, Ferguson B, Dubay C: MonkeySNP. Bioinformatics 2008, 24(22):2645-2646.

doi:10.1186/1471-2164-12-311

Cite this article as: Fawcett et al:: Characterization of single-nucleotide variation in Indian-origin rhesus macaques (Macaca mulatta). BMC Genomics 2011 12:311. 